\title{
Flujo Hídrico no Saturado en Andisoles
}

\author{
Juan Nissen M. ${ }^{1}$, Cristian Quiroz S. ${ }^{1}$, Oscar Seguel S. ${ }^{2}$, Roberto Mac Donald H. ${ }^{1}$ y \\ Achim Ellies Sch. ${ }^{1}$ (In Memoriam) \\ ${ }^{1}$ Instituto de Ingeniería Agraria y Suelos, Facultad de Ciencias Agrarias, Universidad \\ Austral de Chile. Casilla 567, Valdivia, Chile. \\ ${ }^{2}$ Departamento de Ingeniería y Suelos, Facultad de Ciencias Agronómicas, Universidad \\ de Chile. Casilla 1004, Santiago. Correspondenciua: jnissen@uach.cl
}

\section{Non saturated water movement in Andisols}

Key words: Non saturated hydraulic conductivity, water movement, Andisols

\begin{abstract}
The main aim of this research was to determine the unsaturated hydraulic conductivity in three Andisols under two managements, forest and prairie in the Series Bramadero, Pemehue and Osorno, located in the VIIth, IXth and Xth regions of Chile, respectively. Unsaaturated hydraulic conductivity was determined in undisturbed soils a different depths using the methodology of Becher (1970), which combine the measurements of matric potential, continuity law and Darcy's equation. Measuring the soil matric potential for about 95 days the drying process under laboratory conditions was monitored in unaltered soil profiles without water table. Similar conductivities were found in the three soils, with some exceptions by the soil managements and depth. Soils with large pores size diameter distribution (diameter $>10 \mu \mathrm{m}$ ) tended to be higher in unsaturated hydraulic conductivity under low matric potential. However, this values decreased quickly in soils with coarse porosity. Hydraulic conductivity under non saturated condition in the studied Andisols at a matric potential of pF 2.5 fluctuated between $1.9 \times 10-5$ and $3.6 \times 10-5 \mathrm{~cm} \mathrm{~s}-1$ in pasture soils and between 1.4 x 10-5 and $8.1 \times 10-5 \mathrm{~cm} \mathrm{~s}-1$ in forest soils. A homogenous drying process took place when soils were submitted for long period of drought. The drought intensity was slightly greater in soils under pasture compared with forest soils.
\end{abstract}

Palabras clave: Conductividad hidráulica no saturada, movimiento de agua, Andisol. 


\section{RESUMEN}

El objetivo principal de esta investigación fue determinar la conductividad hidráulica en fase no saturada en tres Andisoles bajo dos manejos bosque y praderas en s series Bramadero, Pemehue y Osorno, localizados en la VII ${ }^{\mathrm{a}}$, IX ${ }^{\mathrm{a}}$ y $\mathrm{X}^{\mathrm{a}}$ Regiones de Chile, respectivamente. En muestras de suelo no alteradas se determinó la conductividad hidráulica en fase no saturada a distintas profundidades, mediante la metodología propuesta por Becher (1970), que combina la medición del potencial mátrico en el suelo y la aplicación de las ecuaciones de continuidad y de Darcy. Mediante mediciones de potencial mátrico, se monitoreó durante 95 días en laboratorio el proceso de secado en perfiles de suelo no alterados y en ausencia de un nivel freático artificial. Paralelamente, se determinó la conductividad hidráulica no saturada. La conductividad hidráulica resultó relativamente similar en los tres suelos, con algunas excepciones en cuanto al tipo de uso y profundidad del suelo. En presencia de mayor porosidad de drenaje (diámetro $>10 \mu \mathrm{m}$ ), la conductividad hidráulica en fase no saturada tendió a ser mayor cuando el suelo se encuentra a bajos potenciales mátricos. Sin embargo, ésta cayó rápidamente, debido a una pronta desaturación de esta porosidad gruesa. La conductividad hidráulica no saturada de los Andisoles estudiados a una tensión de $\mathrm{pF} 2,5$ fluctuó en pradera entre $1,9 \times 10^{-5}$ y $3,6 \times 10^{-5} \mathrm{~cm} \mathrm{~s}^{-1} \mathrm{y}$ en bosque entre $1,4 \times 10^{-5}$ y $8,1 \times 10^{-5} \mathrm{~cm}^{*} \mathrm{~s}^{-1}$. Al ser sometidos a un largo periodo de sequía, se produjo un secado profundo y parejo en los suelos. La intensidad de secado fue ligeramente mayor en suelos bajo pradera que bajo bosque.

\section{INTRODUCCIÓN}

La conductividad hidráulica del suelo es una propiedad que se relaciona con el movimiento del agua hacia las raíces de las plantas, la entrada de agua al suelo y el flujo de agua de drenaje, entre otras situaciones (Dirksen, 1999). Las características del suelo que afectan a la conductividad hidráulica son la porosidad total, la distribución de poros, su tamaño y geometría. También afectan las propieaddes del fluido tales como viscosidad y la densidad, además del gradiente hidráulico (Baver y Gardner, 1973). La conductividad hidráulica es una propiedad que caracteriza a los suelos, pero que no siempre constituye una constante, ya que es dependiente de propiedades tales como la agregación y estructura del suelo (Hillel, 1998; Hartge y Horn, 1999). La determinación de la conductividad hidráulica no saturada in situ refleja de mejor forma las condiciones de campo (Dirksen, 1999).

Tanto los Andisoles (trumaos) como los suelos aluviales arenosos, a diferencia de los Ultisoles (rojo arcillosos), tienen una alta conductividad hidráulica a bajas tensiones, debido a su mayor cantidad de macroporos (> 10 um) (Ellies y Vyhmeister, 1981). Lo mismo ocurre en los suelos Placaquand (Ñadis) del sur de Chile (Janssen et al., 2004). A altas tensiones, la conductividad hidráulica de los suelos aluviales arenosos decrece bruscamente y queda por debajo de los Ultisoles y de los Andisoles. Los suelos de texturas medias, como los trumaos, mantienen, aún a altas tensiones, una alta conductividad hidráulica (Ellies y Vyhmeister, 1981). El gradiente hidráulico en los suelos derivados de cenizas volcánicas es pequeño y la conductividad hidráulica en fase no saturada es alta (Ellies y Vyhmeister, 1981). Ellies (1975), citado por Vyhmeister (1980), señala que esta alta conductividad se explica por la gran porosidad de estos suelos y la distribución uniforme de sus poros. También se ha sugerido que la geometría y forma de los poros en estos suelos puede favorecer la conductividad hidráulica. A su vez, esta característica explicaría el por qué estos suelos pierden tan rápidamente el agua y su- 
fren un secado uniforme en profundidad (Vyhmeister, 1980). Según Ellies y Vyhmeister (1981), al comparar dos suelos, (un trumao reciente y un trumao evolucionado), determinaron un gradiente hidráulico muy pequeño, lo que se puede deber a la alta conductividad hidráulica en fase no saturada y al alto porcentaje de poros medianos que presentaron esto suelos. Vyhmeister (1980) encontró valores de $2 \times 10^{-5} \mathrm{~cm} \mathrm{~s}^{-1}$ para Andisoles a pF 2,5. Al comparar los valores de conductividad hidráulica de suelos trumaos y rojo arcillosos con otros suelos de textura similar, se observó que los primeros son marcadamente superiores (Ellies y Vyhmeister, 1981). Los distintos usos y manejos del suelo modifican su estructura; junto con ello, debería cambiar también la conductividad hidráulica saturada y no saturada. La magnitud de estos cambios estructurales depende: i) de la oportunidad y frecuencia de las operaciones de labranza superficial y sub-superficial del suelo y b) del efecto compactador producido por el tránsito de la maquinaria agrícola y/o tránsito de animales (Hartge y Ellies, 1990).

El objetivo de la presente investigación fue estudiar la dinámica del agua en fase no saturada en tres Andisoles de las Regiones
VII ${ }^{a}$, IX $^{\mathrm{a}}$ y $\mathrm{X}^{\mathrm{a}}$ de Chile, sujetos a un manejo de bosque y pradera. Para esto se evaluó el potencial mátrico durante el proceso de secado de los suelos, como asimismo se caracterizó la conductividad hidráulica en fase no saturada a distintas profundidades en el perfil.

\section{MATERIALES Y MÉTODOS}

El estudio se realizó en el Laboratorio de Física de Suelos del Instituto de Ingeniería Agraria y Suelos de la Universidad Austral de Chile entre el mes de septiembre de 2003 y el mes de enero de 2004. Se emplearon tres Andisoles (trumaos), sometidos cada uno a distintos manejos: bosque nativo y pradera permanente.

Se extrajeron muestras de suelo inalterado en cilindros de $\mathrm{PVC}$ de $30 \mathrm{~cm}$ de diámetro por $75 \mathrm{~cm}$ de longitud ( $\sim 53 \mathrm{~L}) \mathrm{sin}$ vegetación. Los cilindros fueron transportados al laboratorio lo más rápidamente posible. Dado el volumen y el peso de cada muestra, no fue posible considerar repeticiones. En un mismo suelo, la distancia máxima entre puntos de extracción para los dos diferentes usos fue de $200 \mathrm{~m}$. En el Cuadro 1, se señala su procedencia y clasificación taxonómica.

CUADRO 1: Suelos analizados y ubicación de la toma de muestras.

TABLE 1: Studied soils and sampling location.

\begin{tabular}{|c|c|c|}
\hline Serie de suelo & Clasificación & Ubicación \\
\hline Bramadero & Humic Haploxerand & $\begin{array}{l}\text { A } 25 \text { km al este de San Clemente hacia } \\
\text { Laguna del Maule, Talca, VIIa Región }\end{array}$ \\
\hline Osorno & Typic Hapludand & $\begin{array}{l}\text { A } 10 \text { km al sur de Osorno, sector Casa de } \\
\text { Lata, Xa Región }\end{array}$ \\
\hline Pemehue & Pachic Fulvudand & $40 \mathrm{~km}$ al suroeste de Villarrica, IXa Región \\
\hline
\end{tabular}

Nota: Los suelos del Cuadro 1 se presentan ordenados de mayor a menor grado de evolución. 
En campo para cada suelo se determinó la distribución del espacio poroso a dos profundidades: 0-15 cm y 15-40 cm, según metodología de Hartge y Horn (1992), usando cilindros de $365 \mathrm{~cm}^{3}$. Para lo anterior se consideraron diez repeticiones, extraídas sobre una superficie de $1 \mathrm{~m}^{2}$.

Los potenciales mátricos que se generaron en los suelos de las columnas se midieron por sensores instalados a diferentes de profundidades. Se utilizaron tensiómetros (marca SDEC, France), provistos de un tubo de PVC tapados con una membrana de silicona. Para la medición, el tensiómetro se conectó a un lector electrónico (sensor de aguja para registrar grado de vacío). El funcionamiento está basado en el uso de una aguja hipodérmica, la que se introduce a través del tapón de silicona y que mide la succión al interior del tubo. Para la preparación de cada tensiómetro, el llenado con agua se hizo de tal forma que quedó un espacio libre entre el nivel de agua en el tubo y el punto inferior del tapón de silicona, para que la aguja esté en contacto con el medio gaseoso. Por otra parte, el potencial mátrico también fue medido con sensores Watermark (Modelo SEN-W5SS) y el medidor electrónico Watermark (Modelo 30KTCD, Spectrum Technologies Inc., USA), que posee lectura digital entre 5 y $200 \mathrm{kPa}$.

Para medir los potenciales mátricos en las muestras de suelo, se procedió a ubicar los tensiómetros con membrana de silicona a $10,15,20,30$ y $40 \mathrm{~cm}$ de profundidad, en tanto que los sensores Watermark fueron ubicados a 10 y $15 \mathrm{~cm}$ de profundidad.

Previo al experimento, los cilindros con muestras fueron colocadas en recipientes con agua, simulando un nivel freático a 60 $\mathrm{cm}$, de tal forma que se mantuvieran lo menos alteradas y conservaran su estado inicial.

Con el equipo descrito, se monitoreó el desarrollo del potencial mátrico durante un período de secado de 95 días, partiendo de un contenido de agua cercano a saturación. Paralelamente, se determinó la variación de la conductividad hidráulica no saturada durante el período de secado de 95 días. Las mediciones se realizaron a intervalos de tiempo de 2, 8, 15, 35, 45, 75 y 95 días, tomando también una medida de la condición inicial. Para el cálculo teórico de la conductividad hidráulica en fase no saturada $\left(\mathrm{K}_{\mathrm{n}}\right)$, se utilizó la metodología descrita por Becher (1970), bajo condiciones de flujo no estacionario, y que corresponde a una combinación de la ecuación de Darcy con la ecuación de continuidad de flujo y cuya expresión final es:

$$
\mathrm{K}_{\mathrm{ns}}=\frac{\Delta L}{\Delta t} \cdot \frac{\overline{\Delta \theta}}{\operatorname{grad} \Psi}
$$

donde $\mathrm{K}_{\mathrm{ns}}=$ Conductividad hidráulica en fase no saturada $\left(\mathrm{cm} \mathrm{s}^{-1}\right) ; \Delta L=$ espacio a recorrer por el agua entre tensiómetros $(\mathrm{cm})$; $\Delta t=$ variación de tiempo (s); $\overline{\Delta \theta}=$ variación promedio del contenido de agua $\left(\mathrm{cm}^{3}\right.$ $\mathrm{cm}^{-3}$ ) en intervalos de tiempo $t_{1} \mathrm{y} t_{2} \mathrm{y}$ grad $\psi=$ gradiente hidráulico $\frac{\Delta \psi}{\Delta L}\left(\mathrm{~cm} \mathrm{~cm}^{-1}\right)$ (Becher, 1970).

\section{RESULTADOS Y DISCUSIÓN}

\section{Distribución de poros y retención de agua de los suelos estudiados}

En el Cuadro 2, se presenta la distribución del espacio poroso para cada suelo, manejo y profundidad $(0-15 \mathrm{~cm}$ y $15-40 \mathrm{~cm})$. Es posible observar, que los sitios bajo bosque presentaron mayor porosidad de drenaje $(>10 \mu \mathrm{m})$. Este mayor espacio poroso debería estar ligado a un mayor grado de estructuración del suelo (Baver y Gardner 1973). El pisoteo superficial en la pradera debería ser la causa de la disminución de la porosidad en comparación con las muestras sub-superficiales. Tanto bajo pradera como bosque, fue posible observar una tendencia a la disminución del espacio poroso en relación con el grado de evolución del suelo. 
CUADRO 2: Valores promedio de distribución de porosidad de los suelos analizados (\%).

TABLE 2: Average values of pore distribution of soils (\%).

\begin{tabular}{|c|c|c|c|c|c|c|}
\hline Serie de suelo & \multicolumn{2}{|c|}{ Bramadero } & \multicolumn{2}{c|}{ Osorno } & \multicolumn{2}{c|}{ Pemehue } \\
\cline { 1 - 6 } $0-15 \mathrm{~cm}$ & Pradera & Bosque & Pradera & Bosque & Pradera & Bosque \\
\cline { 2 - 6 } & & & & & & \\
\cline { 1 - 5 } PDR $>50 \mu \mathrm{m}$ & 6,1 & 13,1 & 9,3 & 23,9 & 7,2 & 18,0 \\
PDL $50-10 \mu \mathrm{m}$ & 8,3 & 12,6 & 10,2 & 13,3 & 12,4 & 12,6 \\
PAU $10-0,2 \mu \mathrm{m}$ & 16,3 & 19,6 & 19,5 & 14,6 & 23,7 & 19,4 \\
PAI $<0,2 \mu \mathrm{m}$ & 26,0 & 19,7 & 24,6 & 22,1 & 23,6 & 22,4 \\
Porosidad total & 56,7 & 65,0 & 63,6 & 73,9 & 66,9 & 72,5 \\
\hline $15-40 \mathrm{~cm}$ & & & & & & \\
PDR $>50 \mu \mathrm{m}$ & 6,9 & 12,5 & 12,5 & 19,1 & 12,0 & 15,3 \\
PDL $50-10 \mu \mathrm{m}$ & 10,9 & 11,4 & 11,2 & 15,4 & 14,6 & 11,7 \\
PAU $10-0,2 \mu \mathrm{m}$ & 20,6 & 18,2 & 18,5 & 21,2 & 23,0 & 27,3 \\
PAI $<0,2 \mu \mathrm{m}$ & 21,3 & 20,2 & 24,0 & 16,4 & 19,6 & 22,1 \\
Porosidad total & 59,7 & 62,4 & 66,2 & 72,1 & 69,2 & 76,4 \\
\hline
\end{tabular}

Nota: Suelos ordenados de mayor a menor grado de evolución. PDR= Poros de drenaje rápido;

$\mathrm{PDL}=$ Poros de drenaje lento ; PAU $=$ Poros de agua útil ; PAI= Poros de agua inútil .

El Cuadro 2 permite ver que los suelos bajo manejo de bosque, tanto en superficie como en profundidad, retuvieron mayores contenidos de agua a bajas tensiones, siendo levemente superiores en las muestras de bosque superficial $(0-15 \mathrm{~cm})$. En relación a los diferentes suelos, Osorno presentó el mayor contenido de porosidad de drenaje en el bosque a los 0-15 cm, seguido por los suelos Pemehue y Bramadero bajo el mismo manejo.

En los suelos bajo pradera ocurrió lo contrario, ya que en todos los suelos el mayor contenido de poros de drenaje correspondió a las muestras sub-superficial. Al comparar los valores de pradera con los de bosque, tanto en superficie como profundidad (15-40 cm), se observó un menor contenido de porosidad de drenaje en los suelos de pradera.

El menor contenido de espacio poroso en los sitios bajo pradera estaría relacionado con la intensidad, frecuencia y edad de la pradera, que generalmente es más intenso y/o degradante en las capas superficiales (Hartge y Ellies, 1990). En el Cuadro 2 se aprecia que en los tres sitios con manejo de pradera, tanto en superficie como en profundidad, dominaron los poros de un diámetro inferior a $10 \mu \mathrm{m}$. Con respecto a los poros $<0,2 \mu \mathrm{m}$, para todas las series de suelo y sus respectivos manejos, su contenido fue alto. En general, los suelos presentaron una distribución homogénea para todos los grupos de poros. Según la distribución de poros en los suelos de bosque, tanto en superficie como en profundidad se podría esperar una mayor conductividad hidráulica no saturada a tensiones bajas (cercana a saturación), comparado con los suelos de pradera, debido al efecto de su porosidad de drenaje. 
El manejo agropecuario puede producir un cambio estructural en el suelo, el cual a su vez debería cambiar la magnitud y dirección de los flujos de agua y aire (Ellies et al., 1997). Al comparar las praderas estudiadas, se podría esperar una mayor velocidad de infiltración en aquellas que presentan un mayor contenido de porosidad de drenaje, mientras que la ascensión capilar debería ser mayor en los suelos que poseen una mayor proporción de poros finos.

\section{Variación del potencial mátrico del suelo al secarse sin nivel freático}

Las columnas de suelo fueron almacenadas en una sala de secado a $25 \mathrm{oC}$ con una humedad relativa cercana al $70 \%$. En general, el secado del suelo bajo estas condiciones fue más lento que el esperado para condiciones naturales, debido a la falta de ventilación y ausencia de consumo vegetal. Una posible explicación adicional al lento secado de estos suelos podría ser que estas muestras pasaron mucho tiempo con un nivel freático y no alcanzaron altos niveles térmicos internamente.

A continuación, se analizará en forma separada los resultados obtenidos para suelos bajo manejo bosque y pradera en las distintas series de suelos.

\section{Suelos bajo bosque}

En las Figuras 1, 2 y 3 es posible observar el desarrollo del proceso de secado en los suelos bajo el manejo de bosque sin presencia de un nivel freático, al ser afectado por un período extenso de sequía de 95 días. Los tres suelos bajo manejo de bosque experimentaron un secado en profundidad levemente diferente. En la serie Osorno-bosque (Figura 1), el secado fue menos continuo a lo largo del perfil, con quiebre a los 15 $\mathrm{cm}$ de profundidad. En cambio las series Pemehue y Bramadero (Figuras 2 y 3 ) experimentaron un secado en profundidad con una mayor continuidad, específicamente a partir del día 8 .

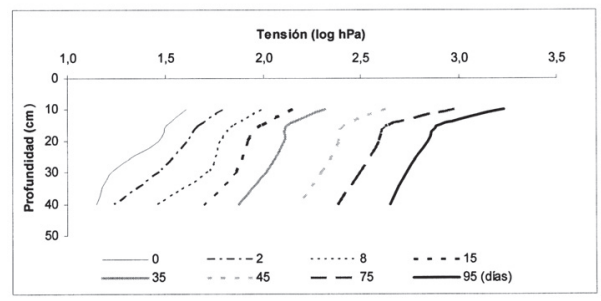

Figura 1.Variación del potencial mátrico durante el secado del suelo Osorno-bosque, sin presencia de nivel freático.

Figure 1. Matric potentials during drying of Osorno forest soil, without water table.

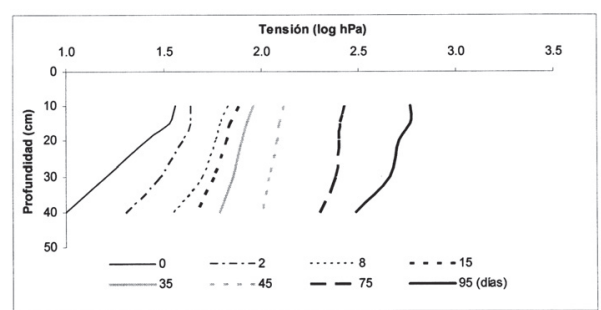

Figura 2. Variación del potencial mátrico durante el secado del suelo pemehue-bosque, sin presencia de nivel freático.

Figure 2. Matric potentials during drying of pemehue forest soil, without water table

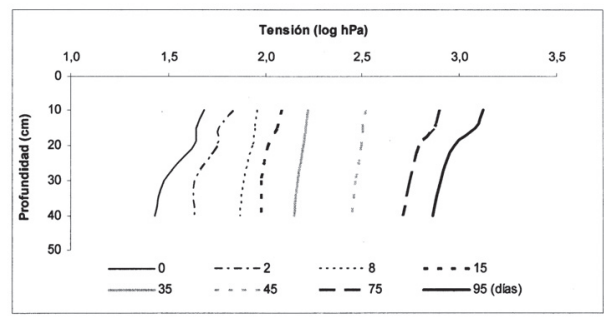

Figura 3. Variación del potencial mátrico durante el secado del suelo bramadero-bosque, sin presencia nivel freático.

Figure 3. Matric potentials during drying of bramadero forest soil, without water table.

Un factor importante en estos suelos fue que en ellos no se formó un colchón anti-evaporante que impidiera un secado en profundidad. Ellies y Vhymeister (1981), al trabajar con suelos Andisoles, señalan que una variación en los valores máximos y mínimos de tensión alcanzados para estos suelos puede deberse a las variaciones de la distribución de poros que sufren los sue- 
los con el humedecimiento y el secado continuo. Según Ellies y Vhymeister (1981), se espera que el suelo que posea el mayor porcentaje de poros medios sufra un mayor secado en profundidad, debido a una mayor conductividad hidráulica no saturada. A partir del Cuadro 2 se puede deducir que, en el suelo Osorno bajo bosque, en promedio para ambas profundidades un $51 \%$ de la porosidad total correspondió a poros menores de $10 \mu \mathrm{m}$, mientras que para los suelos Pemehue y Bramadero fue de $61 \%$. Por lo tanto, la mayor proporción de poros medios y finos en el suelo Bramadero explicaría el secado más homogéneo y más intenso en profundidad que los otros suelos.

$\mathrm{Al}$ apreciar el suelo de Osorno bajo bosque (Figura 1), los gradientes en la capa superficial fueron más pronunciados que los de los suelos Pemehue y Bramadero. Ellies (1975), citado por Vyhmeister (1980), señala que esto puede ocurrir por la mayor cantidad de poros gruesos y por la alta hidrofobía del suelo que afectaría a la conductividad hidráulica, especialmente a medida que las tensiones van en aumento. El efecto estructural del horizonte superficial en este caso funcionaría como un colchón antievaporante.

El secado parejo en profundidad de estos suelos es especialmente probable cuando estos no tienen nivel freático. Como podemos observar en las Figuras 1, 2 y 3, los gradientes superficiales de los suelos fueron más notorios, principalmente para el suelo Osorno bajo bosque. Esto se explicaría, debido a que no hubo una constante reposición del contenido de agua a partir de un nivel freático.

Vyhmeister (1980), señala que un factor que podría favorecer el rápido y constante secado de estos suelos en profundidad es la pérdida por movimiento de vapor de agua. Este tipo de movimiento de agua se ve favorecido por el alto volumen de poros en estos suelos. Estos suelos son muy 'sueltos', lo que permitiría un fácil intercambio gaseoso entre el suelo y la atmósfera.

\section{Suelos bajo pradera}

En las Figuras 4, 5 y 6 podemos apreciar cómo varió el potencial mátrico en el perfil del suelo bajo pradera. Al igual que el caso anterior, se apreció que el secado superficial fue un poco más intenso respecto a las estratas sub-superficiales, especialmente para Pemehue (Figura 5), donde se apreció una diferencia un poco mayor de gradiente entre las estratas superiores y las inferiores. Para los suelos Osorno y Bramadero esta diferencia de gradiente fue menos notoria. La alta proporción de microporos < $0,1 \mu \mathrm{m}$ en las series Bramadero y Osorno (un promedio de $72 \%$ y $67 \%$ de la porosidad total, respectivamente), aseguraría una mayor conductividad hidráulica no saturada, lo que favorecería un secado más uniforme en profundidad. Por otro lado, las tensiones máximas alcanzadas en la serie Pemehue fueron de menor magnitud respecto a los otros suelos. Podemos ver además que el suelo Osorno fue el que alcanzó los valores más altos de tensión al final del período en estudio (Figura 4).

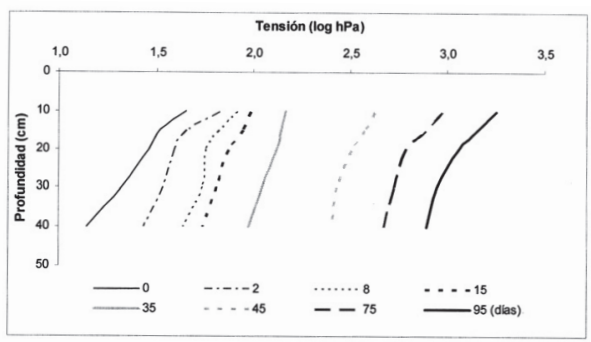

Figura 4. Variación del potencial mátrico durante el secado del suelo osorno- pradera, sin presencia de nivel freático.

Figure 4. Matric potentials during drying of osorno pasture soil, without water table. 


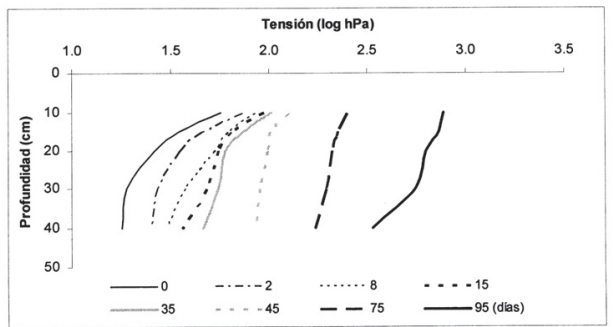

Figura 5.Variación del potencial mátrico durante el secado del suelo pemehue-pradera, sin presencia de nivel freático.

Figure 5. Matric potentials during drying of pemehue pasture soil, without water table.

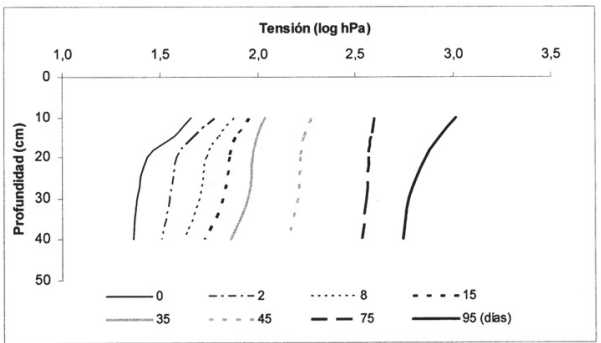

Figura 6. Variación del potencial mátrico durante el secado del suelo

bramadero-pradera, sin presencia de nivel freático.

Figure 6. Matric potentials during drying of bramadero pasture soil, without water table.

Como se mencionó anteriormente, el secado uniforme en profundidad en estos suelos es especialmente probable cuando estos no tienen nivel freático. El gradiente superficial más notorio en el suelo Pemehue podría explicarse porque no existió una constante reposición del contenido de agua desde las estratas inferiores. Otro factor que pudo afectar que no se produzca un rápido y constante secado de este suelo en profundidad pudo ser la escasa pérdida por movimiento de vapor de agua. La pradera de la serie Pemehue presenta una baja proporción de poros de drenaje en superficie en comparación a los otros suelos ( $29 \%$ con poros $>10 \mu \mathrm{m})$, lo que perjudicaría la conducción de vapor. Otro factor que pudo haber favorecido el lento secado de este suelo es que no haya alcanzado altos niveles térmicos, por poseer un alto contenido de agua. Como sabemos, estos suelos son muy 'sueltos' y deberían permitir un fácil intercambio gaseoso entre el suelo y la atmósfera. En general, al realizar una comparación entre los dos manejos considerados en el presente estudio y sin tomar en cuenta el consumo de agua por parte de la vegetación, la intensidad de secado de los suelos fue levemente mayor en pradera que en bosque.

\section{Conductividad hidráulica en fase no saturada}

En las Figuras 7 a y $7 \mathrm{~b}$ se presenta la conductividad hidráulica en fase no saturada para los suelos estudiados (pradera y bosque en el suelo superficial). La conductividad hidráulica en fase no saturada a una misma tensión varió ligeramente en suelos de bosque, no presentando diferencias para suelos bajo pradera. A medida que las tensiones aumentaron, podemos apreciar que la conductividad hidráulica en el suelo superficial bajo pradera fue bastante similar, no observándose diferencias entre ellos (Figura 7a).

Figura $7 \mathrm{a}$ y $7 \mathrm{~b}$
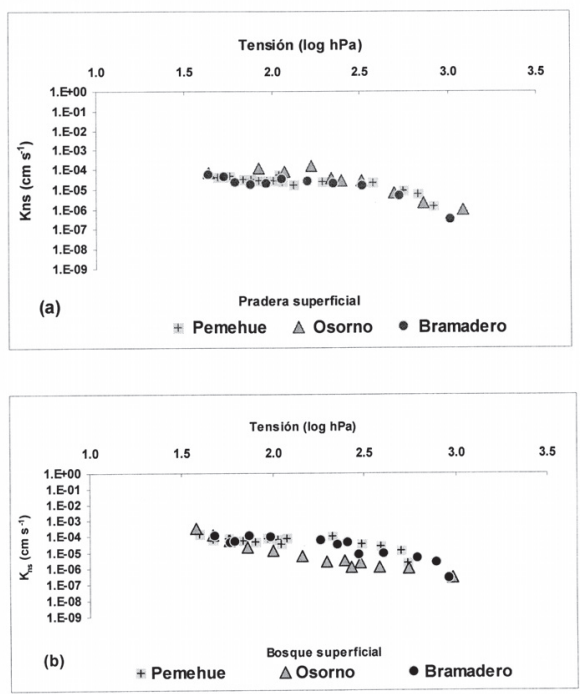

Figura 7. Conductividad hidráulica en fase no saturada (kns) bajo uso de a) pradera y b) bosque. suelo superficial $(0-15 \mathrm{~cm})$.

Figure 7. Non saturated hydraulic conductivity (kns) under: a) pasture and b) forest use. superficial soil $(0-15 \mathrm{~cm})$. 
La serie Osorno bajo pradera a un $\mathrm{pF}$ 2,0 presentó una ligera mayor conductividad, posiblemente por la presencia de ciertos rangos de tamaño de poros. El suelo de bosque Osorno (Figura 7b), debido a su mayor cantidad de poros de drenaje, presentó una mayor conductividad hidráulica a bajas tensiones, pero esta cayó rápidamente al secarse el suelo. Ellies (1975), citado por Vyhmeister (1980), señala que en aquellos rangos de tensiones en los cuales la conductividad hidráulica disminuye o aumenta fuertemente, se estaría indicando la ausencia o presencia, respectivamente de determinados tamaños de poros. En este caso, la conductividad hidráulica de la serie Osorno decreció en mayor medida que los otros suelos, quedando por debajo de los suelos Pemehue y Bramadero. A medida que se secan los suelos y aumentó la fuerza de retención de agua, la conductividad hidráulica descendió bruscamente, cosa que no ocurrió en los suelos de porosidad más fina.

En suelos que presentaron mayor porosidad gruesa $(>10 \mu \mathrm{m})$, a tensiones bajas, la sección media de los poros conductores fue alta, en comparación con suelos que presentaron menor porosidad de este tipo. A medida que estos suelos se secaron los poros más grandes dejaron de conducir el agua. Como los suelos de textura más finas tienen una gran cantidad de poros pequeños, la sección conductora, a tensiones altas es más mayor (Ellies y Vyhmeister, 1981).

Los suelos de textura medias, como en el caso de los Andisoles, mantienen aún a altas tensiones, una alta conductividad hidráulica. Esta alta conductividad se refleja en el secado profundo y parejo en estos suelos en períodos secos (Vyhmeister 1980).

En las Figuras 8 a y $8 b$, se presenta la conductividad hidráulica en fase no saturada de los suelos bajo distinto manejo, para profundidades mayores a $15 \mathrm{~cm}$. Al igual que el caso anterior, se puede apreciar que la conductividad hidráulica en fase no saturada para una misma tensión varió ligeramente entre los suelos.

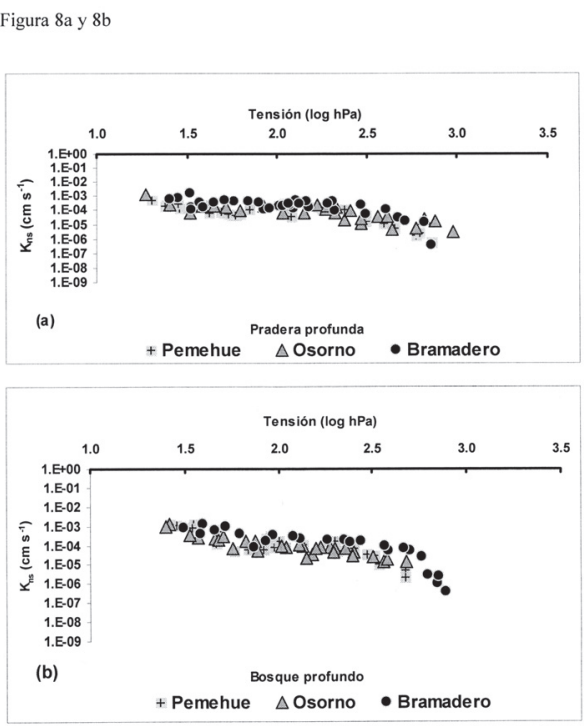

Figura 8. Conductividad hidráulica en fase no saturada (kns) bajo manejo de (a) pradera y (b) bosque. suelo sub-superficial $(15-40 \mathrm{~cm})$.

Figure 8. Non saturated hydraulic conductivity (kns) under a) pasture and b) forest use. subsuperficial soil $(15-40 \mathrm{~cm})$.

Para el rango de tensiones analizadas, la serie Bramadero presentó la mayor conductividad hidráulica en fase no saturada, tanto en pradera como en bosque. Esto podría explicarse porque, a medida que se secan los suelos, los poros más grandes dejan de conducir el agua, sólo la conducen los más pequeños, lo que concuerda con la mayor proporción de poros finos en la serie Bramadero. La alta porosidad de estos suelos y la distribución uniforme de sus poros explican el comportamiento más regular de la conductividad hidráulica en fase no saturada, aunque también se ha sugerido que las formas de los poros en estos suelos puede favorecer la alta conductividad (Ellies y Vyhmeister, 1981).

Al comparar los suelos en superficie con aquellos en profundidad (Figuras 9a, 9b y 9c) se observó, sólo en suelos bajo bosque, la tendencia de una conductividad hidráulica menor en superficie. En los suelos bajo pradera los valores de conductividad hidráulica no saturada también fueron muy 
similares en superficie y en profundidad. Sólo el suelo Bramadero bajo pradera presentó una menor conductividad en superficie, lo que concuerda con los valores bajos de porosidad de drenaje.

Figura $9 a, 9 b$ y $9 c$
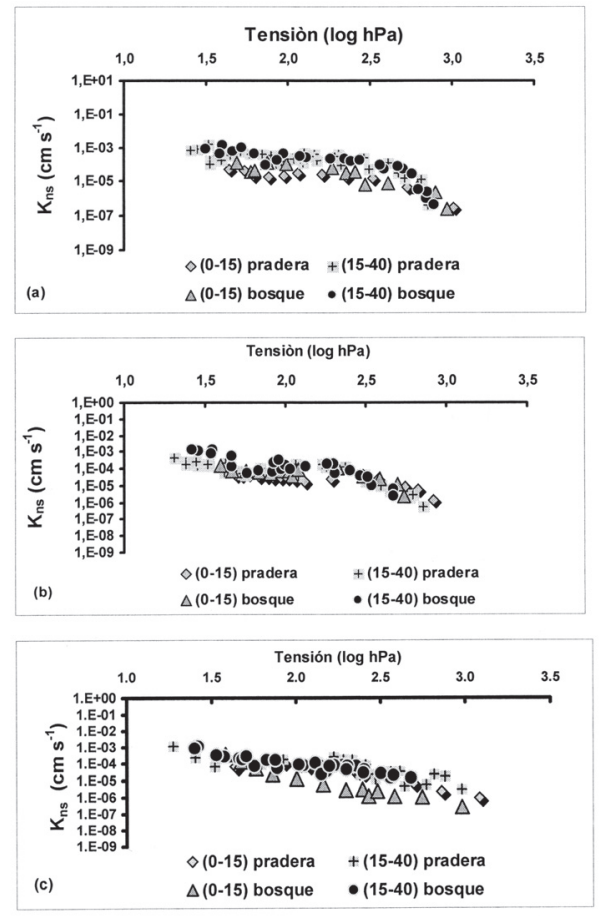

Figura 9. Conductividad hidráulica en fase no saturada (kns) para los suelos (a) bramadero, (b) pemehue y (c) osorno.

Figure 9. Non saturated hydraulic conductivity (kns) of : a) bramadero, (b) pemehue and c) osorno soil.

Al existir una mayor conservación de la estructura en los suelos, se está favoreciendo la conservación del sistema poroso. El mantenimiento de la estructura provoca una conservación de la porosidad secundaria y primaria, las que favorecen los movimientos en fase saturada y no saturada, respectivamente (Ellies et al., 1996). Las afirmaciones de los autores anteriores no se reflejaron claramente en los resultados del presente estudio.
La conductividad hidráulica para los presentes Andisoles a la tensión de pF 2,5 fluctuó en pradera entre $1,9 \times 10^{-5}$ y $3,6 \times$ $10^{-5}$

$\mathrm{cm} \mathrm{s}^{-1}$ y para bosque entre $1,4 \times 10^{-5} \mathrm{y}$ $8,1 \times 10^{-5} \mathrm{~cm} \mathrm{~s}^{-1}$. Estos valores de conductividad hidráulica fueron similares a los detectados por Vyhmeister (1980), quién obtuvo a igual $\mathrm{pF}$ valores de $2 \times 10^{-5} \mathrm{~cm} \mathrm{~s}^{-1}$ con muestras de Andisol.

\section{CONCLUSIONES}

De los resultados del presente estudio se concluyó lo siguiente: Como se esperaba, bajo una vegetación arbórea el espacio poroso del suelo fue mayor que en pradera y dominaron las fracciones de poros de drenaje.

Producto de una conductividad hidráulica relativamente similar en las tres series de suelos estudiadas y sus respectivos manejos (bosque y pradera), se manifestó un secado profundo y parejo en todos los suelos, cuando fueron sometidos a un largo período de sequía por 95 días. Sin considerar el consumo de agua por parte de vegetación original, la intensidad de secado fue levemente mayor en suelos bajo pradera, que aquellos bajo bosque.

Para los distintos suelos y manejos, en presencia de una mayor porosidad de drenaje $(>10 \mu \mathrm{m})$, la conductividad hidráulica en fase no saturada tendió a aumentar cuando el suelo se encontró a bajos potenciales mátricos. Sin embargo, ésta decayó rápidamente, debido a la temprana desaturación de esta porosidad gruesa.

Los gradientes hidráulicos (diferencias de potencial entre estratas superiores e inferiores) fueron más notorios en aquellos suelos que presentaron una distribución menos uniforme de su porosidad en el perfil, lo que explicaría el comportamiento más irregular de su conductividad.

Las conductividades hidráulicas no saturadas de los Andisoles estudiados a una tensión de pF 2,5 fluctuaron en pradera en- 
tre $1,9 \times 10^{-5}$ y $3,6 \times 10^{-5} \mathrm{~cm} \mathrm{~s}^{-1}$ y para bosque entre $1,4 \times 10^{-5}$ y $8,1 \times 10-5 \mathrm{~cm} \mathrm{~s}^{-1}$.

El suelo Bramadero presentó una mayor conductividad hidráulica en muestras subsuperficiales, tanto en bosque como en pradera.

\section{AGRADECIMIENTOS}

Agradecemos al Profesor Francisco Matus, de la Universidad de la Frontera por facilitar los contactos para el muestreo de suelos de la Serie Bramadero en la VII ${ }^{\mathrm{a}} \mathrm{Re}$ gión.

\section{BIBLIOGRAFIA}

BAVER, L.y GARDNER, W.R., 1973. Física de Suelos. Primera edición en español, Ediciones Uteha. México. 525p.

BECHER, H. 1970. Eine Methode zur Messung der Wasserleitfähigkeit von Böden im ungesättigten Zustand. Dissertation. Technische Universität Hannover. 125 p.

DIRKSEN, CH. 1999. Soil physics measurements. Catena Verlag. Reiskirchen. Germany. 154 p.

ELLIES, A. y VYHMEISTER, E., 1981. Algunos aspectos hídricos del horizonte superficial de tres tipos de suelos del sur de Chile. Agro Sur. 9(2): 94-100.

ELLIES, A., GREZ., R., y RAMIREZ, C., 1996. Efecto de la materia orgánica sobre la capacidad de humectación y las propiedades estructurales de algunos suelos de la zona centro sur de Chile. Agro Sur. 24(1): 48-58.
ELLIES, A., GREZ., R. y RAMIREZ, C., 1997. La conductividad hidráulica en fase saturada como herramienta para el diagnóstico de la estructura del suelo. Agro Sur. 25(1): 51-56.

HARTGE, K. H. y ELLIES, A. 1990. Changes in soil structure as caused by land use after clearing virgin forest. Z. f. Bodenkunde und Pflanzenernährung. 153: 389-393.

HARTGE, K. H. y HORN, R.. 1992. Die physikalische Untersuchung von Böden. Eine Labor und Praktikumsanweisung. Ferdinand Enke Verlag. Stuttgart. 168 p.

HARTGE, K. H. y HORN, R. 1999. Einführung in die Bodenphysik. Enke Verlag. Stuttgart. $364 \mathrm{p}$.

HILLEL, D. 1998. Enviromentals soils physics. Academic Press, New York. $771 \mathrm{p}$.

JANSSEN, I., KRUEMMELBEIN, J., HORN, R. y ELLIES, A. 2004. Physical and hydraulic properties of the Nadi-soils in South-Chile. Comparison between untilled and tilled soils. Rev. Ciencia del Suelo y Nutrición Vegetal 4(1):14-28.

VYHMEISTER, E. 1980. Algunos aspectos hídricos del horizonte superficial de tres tipos de suelos del sur de Chile. Tesis Ing. Agr. Valdivia. Universidad Austral de Chile, Facultad de Ciencias Agrarias. 43p. 